\title{
Strong convergence for asymptotically pseudocontractions with the demiclosedness principle in Banach spaces
}

\author{
Yuan-Heng Wang ${ }^{*}$ and Yong-Hui Xia
}

\author{
* Correspondence: \\ wangyuanheng@yahoo.com.cn \\ Department of Mathematics, \\ Zhejiang Normal University, \\ Zhejiang 321004, China
}

\begin{abstract}
The aim of this article is to give an answer to an interesting question proposed in Zhou. At the end of his article, he remarked that it was of great interest to extend his results to certain Banach spaces. So in this article, we extend the demiclosedness principle from Hilbert spaces to Banach spaces. A strong convergence theorem for asymptotical pseudo-contractions in Banach spaces is established. The approaches are based on the extended demiclosedness principle, and the generalized projective operator, and the hybrid method in mathematical programming. Our results extend the previous known results from Hilbert spaces to Banach spaces.
\end{abstract}

MSC: 47H10; 47H09; 47H05.

Keywords: demiclosedness principle, generalized projection, strong convergence, asymptotically pseudo-contractions, Banach space

\section{Introduction}

Zhou [1] proposed an interesting problem at the end of his article. He remarked that it was of great interest to extend his results to certain Banach spaces. Thus, this article essentially pursues two goals.

- The first purpose of this article is to extend the demiclosedness principle from Hilbert spaces to Banach spaces.

- The main aim is to establish a strong convergence theorem for asymptotical pseudo-contractions in Banach spaces. The obtained theorem extends the main result in Zhou [1].

In 1972, Goebel and Kirk [2] introduced the concept of asymptotically nonexpansive mappings in the Hilbert space. Nineteen years later, the class of asymptotical pseudocontraction was introduced by Schu [3]. It is well known that asymptotical nonexpansive mappings form a subclass of asymptotical pseudo-contractions.

Let $H$ be a real Hilbert space with inner product $\langle\cdot, \cdot\rangle, C$ be a nonempty closed convex subset of $H, T$ be a mapping from $C$ into itself, $\left\{k_{n}\right\}$ be a positive real sequence with $k_{n} \rightarrow 1 . T$ is said to be an asymptotical nonexpansive mapping, if the following inequality holds

$$
\left\|T^{n} x-T^{n} y\right\| \leq k_{n}\|x-y\|
$$


for all $x, y \in C$ and all $n \geq 1$. T is called an asymptotical pseudo-contraction if the following inequality holds

$$
\left\langle T^{n} x-T^{n} y, x-y\right\rangle \leq k_{n}\|x-y\|^{2},
$$

for all $x, y \in C$ and all $n \geq 1$.

Further, Schu proved the following convergence theorem in a Hilbert space.

Theorem 1.1 [3] Let $H$ be a Hilbert space; $\phi \neq K \subset H$ closed bounded convex; $L>0 ; T$ : $K \rightarrow K$ completely continuous, uniformly L-Lipschitzian and asymptotically pseudo-contractive with sequence $\left\{k_{n}\right\} \subset[1, \infty) ; q_{n}=2 k_{n}-1$ for all $n \geq 1 ; \sum_{n=1}^{\infty}\left(q_{n}^{2}-1\right)<\infty ;\left\{\alpha_{n}\right\}$, $\left\{\beta_{n}\right\} \subset[0,1] ; \varepsilon \leq \alpha_{n} \leq \beta_{n} \leq b$ for all $n \geq 1$, some $\varepsilon>0$ and some $b \in\left(0, L^{-2}\left[\sqrt{1+L^{2}}-1\right]\right) ; x_{1} \in K$; for all $n \geq 1$, define $x_{n+1}=\left(1-\alpha_{n}\right) x_{n}+\alpha_{n} T^{n} y_{n}, y_{n}=$ $\left(1-\beta_{n}\right) x_{n}+\beta_{n} T^{n} x_{n}$. Then $\left\{x_{n}\right\}$ converges strongly to some fixed points of $T$.

Recently, Zhou [1] extended Schu's results by establishing a fixed point theorem for asymptotically pseudo-contraction without any compact assumption on the mappings. By modifying the algorithm used in Theorem 1.2, Schu successfully proved a strong convergence theorem without any compact assumptions.

Theorem 1.2 [1]Let $C$ be a bounded and closed convex subset of a real Hilbert space $H$. Let $T: C \rightarrow C$ be a uniformly L-Lipschitzian and asymptotical pseudo-contraction with a fixed point. Assume the control sequence $\left\{\alpha_{n}\right\}$ is chosen so that $\alpha_{n} \in[a, b]$ for some $a, b \in\left(0, \frac{1}{1+L}\right)$. Let $\left\{x_{n}\right\}$ be a sequence generated by the following manner

$$
\left\{\begin{aligned}
x_{0} & =x \in C, \\
y_{n} & =\left(1-\alpha_{n}\right) x_{n}+\alpha_{n}+\alpha_{n} T^{n} x_{n \prime} \\
C_{n} & =\left\{z \in C: \alpha_{n}\left[1-(1+L) \alpha_{n}\right]\left\|x_{n}-T^{n} x_{n}\right\|^{2} \leq\left\langle x_{n}-z_{,}\left(y_{n}-T^{n} y_{n}\right)\right\rangle+\left(k_{n}-1\right)(\operatorname{diam} C)^{2}\right\}, \\
Q_{n} & =\left\{z \in C:\left\langle x_{n}-z, x-x_{n}\right\rangle \geq 0,\right\} \\
x_{n+1} & =P_{C_{n} \cap Q_{n}} x, n=0,1,2, \ldots
\end{aligned}\right.
$$

Then the sequence $\left\{x_{n}\right\}$ generated by (1) converges strongly to $P_{F(T)} x$, where $P_{F(T)}$ denotes the metric projection from $H$ onto $F(T)$, a closed convex subset of $H$.

However, all results above are obtained for Hilbert spaces. Motivated by the above mentioned studies, in this article, we first give the concepts of asymptotical pseudocontractions in Banach spaces. Then, we prove the demiclosedness principle in Banach space. Based on our extended demiclosedness principle, we establish a strong theorem for asymptotical pseudo-contractions in Banach spaces. Therefore, we extend the main results of Zhou (see [1]) from Hilbert spaces to Banach spaces. Further, some other results are also improved (see $[4,5])$.

\section{Preliminaries}

This section contains some definitions and lemmas which will be used in the proofs of our main results in the following section.

Throughout this article, let $E$ be a real Banach space and $E^{*}$ be the dual of $E$. The normalized duality mapping $J: E \rightarrow 2^{E *}$ is defined by

$$
J(x)=\left\{f \in E^{*}:\langle x, f\rangle=\|x\|\|f\|,\|x\|=\|f\|\right\}, \forall x \in E,
$$


where $\langle\cdot, \cdot\rangle$ denotes the duality pairing. It is well known (see e.g., [6]) that the operator $J$ is well defined and $J$ is identity mapping if and only if $E$ is a Hilbert space. But in general, $J$ is nonlinear and multiple-valued. So, We have the following definition.

Definition 2.1 The normalized duality mapping $J$ of a Banach space $E$ is said to be weakly sequential continuous, if $\forall\left\{x_{n}\right\} \subset E$, and $x_{n} \rightarrow x$, then there exist $j\left(x_{n}\right) \in J\left(x_{n}\right), j(x)$ $\in J(x)$ such that $j\left(x_{n}\right) \dot{\rightarrow}(x)$, where we denote weak convergence and weak star convergence by $\rightarrow$ and $\rightarrow$ respectively.

Naturally, the concept of asymptotical pseudocontraction can be extended from Hilbert spaces to Banach spaces.

Definition 2.2 Let $C$ be a nonempty closed convex subset of $E$ and let $T$ be a mapping from $C$ into itself. $T$ is said to be an asymptotical pseudocontraction in Banach spaces if there exists a sequence $\left\{k_{n}\right\}$ with $k_{n} \rightarrow 1$ and $j(x-y) \in J(x-y)$ for which the following inequality holds

$$
\left\langle T^{n} x-T^{n} y, j(x-y)\right\rangle \leq k_{n}\|x-y\|^{2},
$$

for all $x, y \in C$ and all $n \geq 1$.

Definition 2.3 [1]A mapping $T: C \rightarrow C$ is said to be uniform L-Lipschitzian if there exists some $L>0$ such that

$$
\left\|T^{n} x-T^{n} y\right\| \leq L\|x-y\|
$$

for all $x, y \in C$ and for all $n \geq 1$.

A Banach space $E$ is said to be strictly convex if $\frac{\|x+y\|}{2}<1$ for $\|x\|=\|y\|=1$ and $x \neq$ $y$; it is also said to be uniformly convex if $\lim _{n \rightarrow \infty},|| x_{n}-y_{n} \|=0$ for any two sequences $\left\{x_{n}\right\},\left\{y_{n}\right\}$ in $E$ such that $\left\|x_{n}\right\|=\left\|y_{n}\right\|=1$ and $\lim _{n \rightarrow \infty} \frac{\left\|x_{n}+y_{n}\right\|}{2}=1$. Let $U=\{x \in E:\|x\|$ $=1\}$ be the unit sphere of $E$, then the Banach space $E$ is said to be smooth provided $\lim _{t \rightarrow 0} \frac{\|x+t y\|-\|x\|}{t}$ exists for each $x, y \in U$. It is also said to be uniformly smooth if the limit is attainted uniformly for each $x, y \in U$. It is well known that if $E$ is reflexive and smooth, then the duality mapping $J$ is single valued. It is also known that if $E$ is uniformly smooth, then $J$ is uniformly norm-to-norm continuous on each bounded subset of $E$. A Banach space $E$ is said to have Kadec-Klee property if a sequence $\left\{x_{n}\right\}$ of $E$ satisfies that $x_{n}$ $\rightarrow x \in E$ and $\left\|x_{n}\right\| \rightarrow\|x\|$, then $x_{n} \rightarrow x$. It is known that if $E$ is uniformly convex, then $E$ has the Kadec-Klee property. Some more properties of the duality mapping have been given in [6,7].

Definition 2.4 [8] Let $E$ be a reflexive and smooth Banach space. The function $\Phi$ : $E \times E \rightarrow R$ is said to be a Lyapunov function defined by

$$
\phi(y, x)=\|y\|^{2}-2\langle y, J x\rangle+\|x\|^{2}
$$

for all $x, y \in E$.

Obviously, we have

(1) $(\|x\|-\|y\|)^{2} \leq \phi(y, x) \leq\left(\|y\|^{2}+\|x\|^{2}\right)$;

(2) $\phi(x, y)=\phi(x, z)+\phi(z, y)+2\langle x-z, J z-J x\rangle$;

(3) $\phi(x, y)=\langle x, J x-J y\rangle+\langle y-x, J y\rangle \leq\|x\|\|J x-J y\|+\|y-x\|\|y\|$, 
for all $x, y, z \in E$.

Lemma 2.1 [8]Let $E$ be a uniformly convex and smooth Banach space, and let $\left\{y_{n}\right\}$, $\left\{z_{n}\right\}$ be two sequences of E. If $\phi\left(y_{n}, z_{n}\right) \rightarrow 0$ and either $\left\{y_{n}\right\}$ or $\left\{z_{n}\right\}$ is bounded, then $y_{n^{-}}$ $z_{n} \rightarrow 0$.

Lemma 2.2 If $E$ is a strictly convex, reflexive, and smooth Banach space, then for $x, y$ $\in E, \phi(x, y)=0$ if and only if $x=y$.

Proof. It is sufficient to show that if $\phi(x, y)=0$ then $x=y$. From (1), we have $\|x\|=$ $\|y\|$. This implies $\langle y, J x\rangle=\|y\|^{2}=\|J x\|^{2}$. From the definition of $J$, we have $J x=J y$. Since $J$ is one-to-one, we have $x=y$.

Definition 2.5 Let $C$ be a closed convex subset of E. Suppose that $E$ is reflexive, strictly convex, and smooth. Then, for any $x \in E$, there exists a point $x_{0} \in C$ such that $\phi\left(x_{0}, x\right)=\min _{y L} \phi(y, x)$. The mapping $P_{C}: E \rightarrow C$ defined by $P_{C} x=x_{0}$ is called the generalized projection $[7,8]$.

The following are well-known results.

Lemma 2.3 [9] Let $C$ be a closed convex subset of a smooth Banach space $E$ and $x L$ E. Then, $x_{0}=P_{C} x$ if and only if

$$
\left\langle x_{0}-y_{,} J x-J x_{0}\right\rangle \geq 0
$$

for all $y \in C$.

Lemma 2.4 [10]Let $E$ be a reflexive, strictly convex, and smooth Banach space and let $C$ be a closed convex subset of $E$ and $x L E$. Then $\phi\left(y, P_{C} x\right)+\phi\left(P_{C} x, x\right) \leq \phi(y, x)$ for all $y \in C$.

\section{Main results}

Theorem 3.1 (Demiclosedness principle) Let E be a reflexive smooth Banach space with a weakly sequential continuous duality mapping J. Let $C$ be a nonempty bounded and closed convex subset of $E$ and $T: C \rightarrow C$ be a uniformly L-Lipschitzian and asymptotical pseudo-contraction. Then I-T is demiclosed at zero, where I is the identical mapping.

Proof. Assume that $\left\{x_{n}\right\} \subset C$ with $x_{n} \rightarrow x$ and $x_{n}-T x_{n} \rightarrow 0$ as $n \rightarrow \infty$; we plan to show that $x \in C$ and $x=T x$. Since $C$ is a closed convex subset of $E, C$ is weakly closed, and hence $x \in C$. So, it is sufficient to show that $x=T x$. To this end, we choose $\alpha \in\left(0, \frac{1}{1+L}\right)$ and define $y_{m}=(1-\alpha) x+\alpha T^{m} x$ for arbitrary but fixed $m \geq 1$. We first show that for fixed $m \geq 1, x_{n}-T^{m} x_{n} \rightarrow 0$, as $n \rightarrow \infty$. In fact, in view of the uniform L-Lipschitz condition of $T$, we have

$$
\left\|x_{n}-T^{m} x_{n}\right\| \leq\left\|x_{n}-T x_{n}\right\|+\left\|T x_{n}-T^{2} x_{n}\right\|+\cdots+\left\|T^{m-1} x_{n}-T^{m} x_{n}\right\| \leq m L\left\|x_{n}-T x_{n}\right\| \rightarrow 0,
$$

as $\mathrm{n} \rightarrow \infty$.

We next estimate $\left\langle J\left(x-y_{m}\right),\left(I-T^{m}\right) y_{m}\right\rangle$. By using the definition of $T$, we have

$$
\begin{aligned}
\left\langle J\left(x-y_{m}\right),\left(I-T^{m}\right) y_{m}\right\rangle= & \left\langle J\left(x-y_{m}\right)-J\left(x_{n}-y_{m}\right),\left(I-T^{m}\right) y_{m}\right\rangle+\left\langle J\left(x_{n}-y_{m}\right),\left(I-T^{m}\right) y_{m}\right\rangle \\
= & \left\langle J\left(x-y_{m}\right)-J\left(x_{n}-y_{m}\right),\left(I-T^{m}\right) y_{m}\right\rangle+\left\langle J\left(x_{n}-y_{m}\right),\left(I-T^{m}\right) x_{n}\right\rangle \\
& +\left\langle J\left(x_{n}-y_{m}\right),\left(I-T^{m}\right) y_{m}-\left(I-T^{m}\right) x_{n}\right\rangle \\
\leq & \left.\left\langle J\left(x-y_{m}\right)-J\left(x_{n}-y_{m}\right),\left(I-T^{m}\right) y_{m}\right)\right\rangle+\left\langle J\left(x_{n}-y_{m}\right),\left(I-T^{m}\right) x_{n}\right\rangle \\
& +\left(k_{m}-1\right)\left\|y_{m}-x\right\|^{2} .
\end{aligned}
$$


Since $x_{n} \rightarrow x$ by our assumption and $x_{n}-T x_{n} \rightarrow 0$ as $\mathrm{n} \rightarrow \infty$, by $x_{n}-T^{m} x_{n} \rightarrow 0$, as $\mathrm{n} \rightarrow \infty$ and since $J$ is a weakly sequential continuous duality mapping, it follows that

$$
\left\langle J\left(x-y_{m}\right),\left(I-T_{m}\right) y_{m}\right\rangle \leq\left(k_{m}-1\right)(\operatorname{diam} C)^{2},
$$

as $\mathrm{n} \rightarrow \infty$

Further, using the uniform L-Lipschitz condition of $T$ and the definition of $y_{m}$, we have

$$
\left\langle J\left(x-y_{m}\right),\left(I-T^{m}\right) x-\left(I-T^{m}\right) y_{m}\right\rangle \leq(1+L)\left\|x-y_{m}\right\|^{2} \leq(1+L) \alpha^{2}\left\|x-T^{m} x\right\|^{2} .
$$

At this point, by the facts above, we have

$$
\begin{aligned}
\left\|x-T^{m} x\right\|^{2} & =\left\langle J\left(x-T^{m} x\right), x-T^{m} x\right\rangle=\frac{1}{\alpha}\left\langle J\left(x-y_{m}\right), x-T^{m} x\right\rangle \\
& =\frac{1}{\alpha}\left\langle J\left(x-y_{m}\right), x-T^{m} x-\left(y_{m}-T^{m} y_{m}\right)\right\rangle+\frac{1}{\alpha}\left\langle J\left(x-y_{m}\right), y_{m}-T^{m} y_{m}\right\rangle \\
& \leq \alpha(1+L)\left\|x-T^{m} x\right\|^{2}+\frac{1}{\alpha}\left\langle J\left(x-y_{m}\right), y_{m}-T^{m} y_{m}\right\rangle \\
& \leq \alpha(1+L)\left\|x-T^{m} x\right\|^{2}+\frac{1}{\alpha}\left(k_{m}-1\right)(\operatorname{diam} C)^{2}
\end{aligned}
$$

which implies that

$$
\alpha[1-\alpha(1+L)]\left\|x-T^{m} x\right\|^{2} \leq\left(k_{m}-1\right)(\operatorname{diam} C)^{2}
$$

for all $m \geq 1$.

Letting $m \rightarrow \infty, k_{m} \rightarrow 1$ yields that $T^{m} x \rightarrow x$, and hence $T^{m+1} x \rightarrow T x$ as $m \rightarrow \infty$, since $T: C \rightarrow C$ is continuous. Consequently, we have $x=T x$, completing the proof of Theorem 3.1.

Remark 3.1 Theorem 3.1 is useful in Banach spaces and a novel result which will play a very key role for establishing the strong convergence theorem of fixed points of asymptotical pseudo-contractions in this article.

Theorem 3.2 Let $E$ be a uniformly convex and uniformly smooth Banach space with a weakly sequential continuous convex duality mapping J. Let $C$ be a nonempty bounded and closed convex subset of $E$, and let $T: C \rightarrow C$ be a uniform L-Lipschitzian, convex and asymptotical pseudo-contraction with $F(T)$ nonempty. Assume the control sequence $\left\{\alpha_{n}\right\}$ is chosen so that $\alpha_{n} \in[a, b]$ for some $a, b \in\left(1+\frac{1}{1+L}\right)$. The sequence $\left\{x_{n}\right\}$ is given in the following manner

$$
\left\{\begin{aligned}
x_{0} & =x \in C, \\
y_{n} & =\left(1-\alpha_{n}\right) x_{n}+\alpha_{n} T^{n} x_{n} \\
H_{n} & =\left\{z \in C: \alpha_{n}\left[1-(1+L) \alpha_{n}\right]\left\|x_{n}-T^{n} x_{n}\right\|^{2},\right. \\
& \left.\leq\left\langle J\left(x_{n}-y_{n}\right)-J\left(z-y_{n}\right),\left(y_{n}-T^{n} y_{n}\right)\right\rangle+\left(k_{n}-1\right)(\operatorname{diam} C)^{2}\right\}, \\
W_{n} & =\left\{z \in C:\left\langle x_{n}-z, J x-J x_{n}\right\rangle \geq 0\right\}, \\
x_{n+1} & =P_{H_{n} \cap W_{n}} x, n=0,1,2, \ldots
\end{aligned}\right.
$$

Then the sequence $\left\{x_{n}\right\}$ converges strongly to $P_{F(T)} x$, where $P_{F(T)}$ is the generalized projection from $E$ onto $F(T)$.

Proof. We divide the proof into seven steps.

Step 1. $P_{F(T)}$ is well defined for every $x \in C$. 
Since $T$ is uniform L-Lipschitzian continuous and convex, we know $F(T)$ is closed and convex. Moreover, $F(T)$ is nonempty by our assumption. Therefore, $P_{F(T)}$ is well defined for every $x \in C$.

Step 2 Show that $H_{n}$ and $W_{n}$ are closed and convex for all $n \geq 0$.

From the definitions of $W_{n}$ and $H_{n}$, it is obvious that $W_{n}$ is closed and convex and $H_{n}$ is closed for each $n \geq 0 . H_{n}$ is convex for each $n \geq 0$, which follows from the convexity of $J$. We omit the details.

Step 3. Show that $F(T) \subset H_{n} \cap W_{n}$ for all $n \geq 0$.

We first prove $F(T) \subset H_{n}$. Let $u \in F$ and let $n \geq 0$. Then, using (2), the uniform LLipschitz continuity of $T$ and the asymptotical pseudo-contractiveness of $T$, we have

$$
\begin{aligned}
\left\|x_{n}-T^{n} x_{n}\right\|^{2} & =\left\langle J\left(x_{n}-T^{n} x_{n}\right), x_{n}-T^{n} x_{n}\right\rangle=\frac{1}{\alpha_{n}}\left\langle J\left(x_{n}-y_{n}\right), x_{n}-T^{n} x_{n}\right\rangle \\
& =\frac{1}{\alpha_{n}}\left\langle J\left(x_{n}-y_{n}\right), x_{n}-T^{n} x_{n}-\left(y_{n}-T^{n} y_{n}\right)\right\rangle+\frac{1}{\alpha_{n}}\left\langle J\left(x_{n}-y_{n}\right), y_{n}-T^{n} y_{n}\right\rangle \\
& \leq \alpha_{n}(1+L)\left\|x-T^{m} x\right\|^{2}+\frac{1}{\alpha_{n}}\left\langle J\left(x_{n}-y_{n}\right)-J\left(p-y_{n}\right), y_{n}-T^{n} y_{n}\right\rangle \\
& +\frac{1}{\alpha_{n}}\left\langle J\left(p-y_{n}\right), y_{n}-T^{n} y_{n}-\left(p-T^{n} p\right)\right\rangle \\
& \leq \alpha_{n}(1+L)\left\|x_{n}-T^{n} x_{n}\right\|^{2}+\frac{1}{\alpha_{n}}\left(k_{n}-1\right)(\operatorname{diam} C)^{2} \\
& +\frac{1}{\alpha_{n}}\left\langle J\left(x_{n}-y_{n}\right)-J\left(p-y_{n}\right), y_{n}-T^{n} y_{n}\right\rangle,
\end{aligned}
$$

which implies that

$$
\alpha_{n}\left[1-\alpha_{n}(1+L)\right]\left\|x_{n}-T^{n} x_{n}\right\|^{2} \leq\left(k_{n}-1\right)(\operatorname{diam} C)^{2}+\left\langle J\left(x_{n}-y_{n}\right)-J\left(p-y_{n}\right), y_{n}-T^{n} y_{n}\right\rangle,
$$

and which shows that $u \in H_{n}$ for all $n \geq 0$. This proves that $F(T) \subset H_{n}$ for all $n \geq 0$. Next we prove $F(T) \subset W_{n}$ for all $n \geq 0$ by induction. For $n=0$, we have $F(T) \subset C=$ $W_{0}$. Assume that $F(T) \subset W_{n}$. Since $x_{n+1}$ is the projection of $x$ onto $H_{n} \cap W_{n}$, by Lemma 2.3, we have

$$
\left\langle x_{n+1}-z, J x-J x_{n+1}\right\rangle \geq 0,
$$

for any $z \in H_{n} \cap W_{n}$. As $F(T) \subset H_{n} \cap W_{n}$ by the induction assumption, the last inequality holds, in particular, for all $u \in F(T)$. This together with the definition of $W_{n+1}$ implies that $F(T) \subset W_{n+1}$. Hence, $F(T) \subset H_{n} \cap W_{n}$ for all $n \geq 0$.

Step 4. $\left\|x_{n+1}-x_{n}\right\| \rightarrow 0$ as $n \rightarrow \infty$.

In view of (2) and Lemma 2.3, we have $x_{n}=P_{W_{n}} x$, which means that for any $z \in W_{w}$ $\phi\left(x_{w}, x\right) \leq \phi(z, x)$. Since $x_{n+1} \in W_{n}$ and $u \in F(T) \subset W_{n}$, we obtain

$$
\phi\left(x_{n}, x\right) \leq \phi\left(x_{n+1}, x\right) \operatorname{and} \phi\left(x_{n}, x\right) \leq \phi(u, x),
$$

for all $n \geq 0$. Consequently, $\lim _{n \rightarrow \infty} \phi\left(x_{n}, x\right)$ exists and $\left\{x_{n}\right\}$ is bounded. By using Lemma 2.4, we have

$$
\phi\left(x_{n+1}, x_{n}\right) \leq \phi\left(x_{n+1}, x\right)-\phi\left(x_{n}, x_{n}\right) \rightarrow 0,
$$

as $n \rightarrow \infty$. By using Lemma 2.1, we obtain $\left\|x_{n+1}-x_{n}\right\| \rightarrow 0$ as $n \rightarrow \infty$.

Step 5. $\left\|x_{n}-T^{n} x_{n}\right\| \rightarrow 0$ as $n \rightarrow \infty$. 
It follows from step 4 that $\left\|x_{n+1}-x_{n}\right\| \rightarrow 0$ as $n \rightarrow \infty$. Since $x_{n+1} \subset H_{n}$, noting that $\left\{\alpha_{n}\right\}$ is chosen so that $\alpha_{n} \in[a, b]$ for some $a, b \in\left(1+\frac{1}{1+L}\right),\left\{y_{n}\right\}$ and $\left\{T^{n} y_{n}\right\}$ are bounded and $J$ is a weakly sequential continuous duality mapping, from the definition of $H_{n}$, we have

$$
\begin{aligned}
\alpha_{n}\left[1-\alpha_{n}(1+L)\right]\left\|x_{n}-T^{n} x_{n}\right\|^{2} & \leq\left(k_{n}-1\right)(\operatorname{diam} C)^{2}+\left\langle J\left(x_{n}-y_{n}\right)-J\left(p-y_{n}\right), y_{n}-T^{n} y_{n}\right\rangle \\
& \leq\left(k_{n}-1\right)(\operatorname{diam} C)^{2}+\left\|J\left(x_{n}-y_{n}\right)-J\left(p-y_{n}\right)\right\|\left\|y_{n}-T^{n} y_{n}\right\| \rightarrow 0,
\end{aligned}
$$

as $n \rightarrow \infty$.

Step 6. $\left\|x_{n}-T x_{n}\right\| \rightarrow 0$, as $n \rightarrow \infty$.

Observing that

$$
\begin{aligned}
\left\|x_{n}-T x_{n}\right\| & \leq\left\|x_{n}-x_{n+1}\right\|+\left\|x_{n+1}-T^{n+1} x_{n+1}\right\|+\left\|T^{n+1} x_{n+1}-T^{n+1} x_{n}\right\|\left\|T^{n+1} x_{n}-T x_{n}\right\| \\
& \leq\left\|x_{n}-x_{n+1}\right\|+\left\|x_{n+1}-T^{n+1} x_{n+1}\right\|+L\left\|x_{n}-x_{n+1}\right\|+L\left\|T^{n} x_{n}-x_{n}\right\| \\
& \leq(1+L)\left\|x_{n}-x_{n+1}\right\|+\left\|x_{n+1}-T^{n+1} x_{n+1}\right\|+L\left\|T^{n} x_{n}-x_{n}\right\|
\end{aligned}
$$

and using steps 4 and 5, we reach the desired conclusion.

Step 7. $x_{n} \rightarrow P_{F(T)} x$, as $n \rightarrow \infty$.

From the result of step 6, we know that if $\left\{x_{n_{k}}\right\}$ is a subsequence of $\left\{x_{n}\right\}$ such that $\left\{x_{n_{k}}\right\} \rightarrow \hat{x} \varepsilon C$, then by the Theorem 3.1, we obtain $\hat{x} \in F(T)$. So we assume $\left\{x_{n_{k}}\right\}$ be a subsequence of $\left\{x_{n}\right\}$ such that $\left\{x_{n_{k}}\right\} \rightarrow \hat{x} \in F(T)$ and $\omega=P_{F(T)}$. For any $n \geq 1$, from $x_{n+1}=P_{H_{n \cap W_{n}}} x$ and $\omega \in F(T) \subset H_{n} \cap W_{n}$, we have $\phi\left(x_{n+1}, x\right) \leq \phi(\omega, x)$.

On the other hand, from the weak lower semicontinuity of the norm, we have

$$
\begin{aligned}
\phi(\hat{x}, x) & =\|\hat{x}\|^{2}-2\langle\hat{x}, J x\rangle+\|x\|^{2} \\
& \leq \liminf _{n \rightarrow \infty}\left(\left\|x_{n_{k}}\right\|^{2}-2\left\langle\left\|x_{n_{k}}\right\|^{2}, J x\right\rangle+\|x\|^{2}\right) \\
& =\liminf _{n \rightarrow \infty} \phi\left(x_{n_{k}}, x\right) \leq \limsup _{n \rightarrow \infty} \phi\left(x_{n_{k}}, x\right) \leq \phi(\omega, x) .
\end{aligned}
$$

From the definition of $P_{F(T)}$, we obtain $\hat{x}=\omega$ and hence $\lim _{n \rightarrow \infty} \phi\left(x_{n_{k}}, x\right)=\phi(\omega, x)$. So we have $\lim _{k \rightarrow \infty}\left\|x_{n_{k}}\right\|=\|\omega\|$. Using the Kadec-klee property of $E$, we obtain that $\left\{x_{n_{k}}\right\}$ converges strongly to $P_{F(T)} x$. Since $\left\{x_{n_{k}}\right\}$ is an arbitrary weakly convergent sequence of $\left\{x_{n}\right\}$, we can conclude that $\left\{x_{n}\right\}$ converges strongly to $P_{F(T)} x$.

Remark 3.2 Theorem 3.2 extends the main results of Zhou (see [1]) from Hilbert spaces to Banach spaces and improves some other results (see [4,5]). Moreover, our method used in this article can be applied to other mappings, such as $k$-strict pseudocontractions (see [11]) for some $k \in[0,1)$ in Banach spaces. It should be pointed out that our extended demiclosed-ness principle plays a key role in the proof.

\section{Acknowledgements}

The authors would like to thank editors and referees for many useful comments and suggestions for the improvement of the article. This study was supported by the National Natural Science Foundations of China (Grant Nos. 11071169 and 10901140), the Natural Science Foundations of Zhejiang Province of China (Grant No. Y6100696) and Zhejiang Innovation Project (Grant No. T200905).

\section{Authors' contributions}

All authors contributed equally and significantly in this research work. All authors read and approved the final manuscript.

\section{Competing interests}

The authors declare that they have no competing interests. 


\section{References}

1. Zhou, HY: Demiclosedness principle with applications for asymptotically pseudo-contractions in Hilbert spaces. Nonlinear Anal. 70, 3140-3145 (2009)

2. Goebel, K, Kirk, WA: A fixed point theorem for asymptotically nonexpansive mappings. Proc Am Math Soc. 35, 171-174 (1972)

3. Schu, J: Iterative construction of fixed points of asymptotically nonexpansive mappings. J Math Anal Appl. 158, 407-413 (1991)

4. Liu, QH: Convergence theorems of the sequence of iterates for asymptotically demicon-tractive and hemicontractive mappings. Nonlinear Anal. 26, 1835-1842 (1996)

5. Kim, TH, Xu, HK: Strong convergence of modified Mann iterations for asymptotically nonexpansive mappings and semigroups. Nonlinear Anal. 64, 1140-1152 (2006)

6. Cioranescu, I: Geometry of Banach Spaces. Duality Mappings and Nonlinear Problems. Kluwer Academic Publishers Dordrecht (1990)

7. Li, J: The generalized projection operator on reflexive Banach spaces and its applications. J Math Anal Appl. 306, 55-71 (2005)

8. Kamimura, S, Takahashi, W: Strong convergence of a proximal-type algorithm in a Banach space. SIAM J Optim. 13, 938-945 (2002)

9. Alber, Yal: Metric and generalized projection operators in Banach spaces: properties and applications. In: Kartsatos AG (ed.) Theory and Applications of Nonlinear Op-erator of Accretive and Monotone Type,Lecture Notes in Pure and Appl Math, vol. 178, pp. 15-50. Marcel Dekker New York (1996)

10. Wang, YH, Zeng, LC: Convergence of generalized projective modified iterative methods in Banach spaces (in Chinese) Chin Ann Math. 30A, 55-62 (2009)

11. Ceng, LC, Al-Homidan, S, Ansari, QH, Yaod, JC: An iterative scheme for equilibrium problems and fixed point problems of strict pseudo-contraction mappings. J Comput Appl Math. 223, 967-974 (2009)

doi:10.1186/1687-1812-2012-45

Cite this article as: Wang and Xia: Strong convergence for asymptotically pseudocontractions with the demiclosedness principle in Banach spaces. Fixed Point Theory and Applications 2012 2012:45.

\section{Submit your manuscript to a SpringerOpen ${ }^{\circ}$} journal and benefit from:

- Convenient online submission

Rigorous peer review

- Immediate publication on acceptance

- Open access: articles freely available online

- High visibility within the field

- Retaining the copyright to your article

Submit your next manuscript at $>$ springeropen.com 